\title{
Beneficios de la protocolización en la atención de los enfermos con insuficiencia cardíaca
}

\author{
Manuel Lorenzo López Reboiro', José Manuel Cerqueiro González² \\ ${ }^{1}$ Servicio de Medicina Interna. Hospital Comarcal de Monforte de Lemos. ${ }^{2}$ Servicio de Medicina Interna. Hospital Lucus Augusti
}

En la actualidad, el esfuerzo del sistema sanitario y de los profesionales que lo componen está centrado en controlar la pandemia producida por la COVID 19. El año 2020 será recordado por el impacto de esta pandemia y el año actual parece ir en la misma línea. La sobrecarga asistencial fue evidente y los profesionales tuvieron que volcarse rápidamente en atender la elevada demanda, dejando temporalmente de lado otra atención menos urgente y la mayoría de la actividad programada. En el transcurrir de las diferentes olas pandémicas además de ingresar pacientes con COVID 19 también ingresaron pacientes por patologías crónicas descompensadas, por lo que los internistas se encontraron con dos frentes abiertos, el de la enfermedad emergente (COVID19) y el de la patología crónica. Esta presión asistencial continuada ha desgastado a los profesionales y sobrecargado al sistema, por lo que se vuelve más importante que nunca la correcta gestión de los recursos disponibles.

Si valoramos el impacto de las diversas patologías crónicas, las Enfermedades Circulatorias siguen siendo la causa más frecuente de ingreso en mayores de 65 años y la primera causa de muerte según el Instituto Nacional de Estadística (INE), y dentro de este grupo de enfermedades la Insuficiencia Cardíaca (IC) ocupa el primer lugar ${ }^{1}$. Además, en el Registro RECALMIN realizado en el 2016, el diagnóstico principal más prevalente en las altas de Medicina Interna fue IC. Estos datos nos orientan cuán importante es la carga asistencial creada por los pacientes con IC y la necesidad de reducir ingresos y mejorar la supervivencia².

Para mejorar la calidad asistencial a estos pacientes, contamos con un arsenal terapéutico, que crece continuamente, aunque la mayor parte de estas medidas están orientadas a paciente con IC con fracción de eyección deprimida. Entre los avances más destacados tenemos la mejora en técnica invasivas, el descubrimiento de nuevos fármacos que modifican la historia natural de la enfermedad y la mejora en el tratamiento de las comorbilidades. Además, dado que el futuro del paciente está marcado por episodios de descompesación, es necesario promover programas asinstenciales que abarquen toda la vida del paciente, integrando el manejo de sus comorbilidades, coordinando la atención primaria con la hospitalaria y implicando al propio paciente y a sus cuidadores ${ }^{3}$. Con el objetivo de promover una medicina más protocolizada, y de que todos los profesionales hagamos una atención más uniforme, independiente del centro donde se reciba o de la persona que la ejerza, tenemos una serie de herramientas a nuestro alcance como son las guías de práctica clínica y las recomendaciones de las Sociedades Científicas.

Las guías de práctica clínica son ampliamente conocidas y utilizadas. En ellas se agrupan las posibles intervenciones que podemos hacer sobre el paciente adjudicándoles un grado de evidencia científica a partir de la bibliografía existente en ese momento, por lo tanto necesitan inexorablemente de una actualización constante. El internista debe conocer estas guías y adherirse a ellas en la medida de lo posible, entendiendo que los pacientes que maneja, son más ancianos y pluriopatológicos que aquellos que formaron las diferentes cohortes de los ensayos clínicos. Por lo tanto, los efectos secundarios, los efectos adversos, la intolerancia, etc. suelen ser más frecuentes.

Las recomendaciones que exponemos a continuación son las de la SEMI y SOGAMI, dentro de éstas existen grupos de trabajo de Insuficiencia Cardíaca y Fibrilación Auricular que promueven la interacción entre profesionales que se dedican especialmente a este tema, con el objetivo de aunar criterios y crear el ambiente necesario para que se generen nuevos campos de trabajo e investigación. Dentro de los proyectos llevados a cabo en estos grupos cabe mencionar cuatro: Manual Práctico de Manejo Integral del paciente con Insuficiencia Cardíaca Crónica, Protocolo de Atención de la Insuficiencia Cardíaca Aguda, Programa de Atención Integral de Pacientes con Insuficiencia Cardíaca (Programa UMIPIC), y el Protocolo de Atención a la Insuficiencia Cardíaca Crónica.

El manual práctico de Manejo Integral del Paciente con Insuficiencia Cardíaca es una guía ampliamente conocida, dirigida por el Dr Luis Manzano, estando actualmente en realización la $5^{a}$ edición. Se trata de un manual fundamentalmente práctico, que además de centrarse en el manejo de la IC, aborda el abanico de comorbilidades más frecuentes que presentan estos pacientes, con capítulos muy interesantes e imprescindibles como el manejo del síndrome de resistencia a diuréticos o el tratamiento con IC refractaria o paliativa ${ }^{4}$.

El Protocolo de Atención de Insuficiencia Cardíaca Aguda nace en el 2021 como un "Checklist" para protocolizar la atención a los paciente con IC desde el momento en que ingresan hasta el momento del alta. Este documento se estructura en cuatro partes: consideraciones durante el ingreso, manejo de la fase con- 


\section{Si valoramos el impacto de las diversas patologías crónicas,}

las Enfermedades Circulatorias siguen siendo la causa más frecuente de ingreso en mayores de 65 años y la primera causa de
muerte según el Instituto Nacional de Estadística (INE)

gestiva, manejo de la fase estable y las consideraciones al alta. Las consideraciones al ingreso se centran en la tipificación de la cardiopatía y comorbilidades, situación clínica del paciente y desencadenante. En el manejo de la fase congestiva orienta a las opciones de soporte hemodinámico, ventilatorio y diurético. La fase estable se centra en el ajuste de tratamientos que modifiquen la historia natural de la enfermedad, control de comorbilidades y educación del paciente. Por último, las consideraciones antes del alta estratifican el riesgo del paciente para poder programar la transición de la hospitalización al control ambulatorio ${ }^{5}$.

El Protocolo de Atención de Insuficiencia Cardíaca Crónica se creó en el 2021 con el objetivo de ser una guía para la atención ambulatoria de los pacientes con IC. En un formato similar al protocolo anterior, establece un "cheklist" para los escenarios de la práctica clínica habitual como son: primera consulta, consulta programada, consulta no programada, consulta post alta, coordinación con primaria y otros especialistas, y coordinación con Unidades de IC avanzada y paliativos. Además en este documento podemos encontrar anexos con algoritmos diagnósticos y terapéuticos. Por último, no solo orienta a los médicos internistas sino que también aborda el papel de la consulta de enfermería, considerada pieza clave para la detección precoz de descompensaciones y el seguimiento continuado de estos pacientes, desempeñando el papel de educador y vertebrando la consulta ${ }^{6}$.

Para terminar, mencionar el Programa UMIPIC, un modelo de consulta monográfica de IC en la que un Especialista en Medicina Interna y un Profesional de Enfermería dan cobertura integral, intensiva y protocolizada a pacientes pluripatológicos no candidatos a tratamientos invasivos, con buen soporte familiar y sin deterioro cognitivo grave. En esta consulta se hace un seguimiento integral de todas las patologías médicas del paciente además de su IC. La atención se organiza en torno a numerosas consultas protocolizadas de médico y de enfermería, incluyendo consultas no programadas para actuar precozmente ante una descompensación. Para llegar a este punto es necesario por un lado educar en la enfermedad a los pacientes y/o cuidadores, y por otro contar con recursos y personal médico y de enfermería preparado. Además es muy importante que exista un circuito que permita la cooperación con otros especialistas y atención primaria ${ }^{7}$.

Este modelo de consulta se promociona desde la
SEMI y la SOGAMl a través de sus grupos de trabajo desde el año 2011. En la actualidad hay un total de 34 consultas UMIPIC en España y 5 en Galicia. Los datos de los pacientes atendidos en estas unidades se registran a través de la plataforma RICA, lo que permitió demostrar el impacto positivo de este modelo en cuanto a la reducción de hospitalizaciones y aumento de la supervivencia ${ }^{8,9}$.

En conclusión, actualmente el sistema sanitario se ve sometido a una importante presión asistencial por la aparición de nuevas patologías. Pero a pesar de ello hay que seguir mejorando e implementando cambios que nos ayuden a reducir ingresos por otras patologías tan prevalentes como la IC. En esta patología en particular, destacamos la importancia de la protocolización de la asistencia y la implantación de programas de seguimiento como la consulta modelo UMIPIC. La reducción de ingresos y complicaciones derivadas de estas patologías ahondará en una mejoría en la calidad de vida de los pacientes y en una mejor gestión de los recursos hospitalarios.

\section{BIBLIOGRAFÍA}

1. Instituto nacional de estadística. Encuestas de morbilidad hospitalaria. Disponible en: https://www.ine.es/dyngs/INEbase/es/operacion.htm?c=Estadistica_C\&c $i \mathrm{i}=1254736176778 \&$ menu $=u$ ultiDatos\& $i \mathrm{~d}=1254735573175$. Comprobado $31 / 08 / 2021$

2. Instituto nacional de estadística. Estadísticas de defunciones según causa de muerte. Disponible en: https://www.ine.es/dyngs/NEbase/es/operacion.htm?c= Estadistica_C\&cid=1254736176780\&menu=ultiDatos\&idp $=1254735573175$. Comprobado 31/08/2021

3. A. Zapatero Gaviria, R. Barba Martin, P RomanSanchez, E Casariego Vales, J Diez Manglano, M GarciaCors. RECALMIN. LA atención al paciente en las unidades de Medicina Interna del Sistema Nacional de Salud. Revista clínica española: publicación oficial de la Sociedad Española de Medicina Interna. 2016; 216, 175-182.

4. Theresa A. McDonagh, Marco Metra, Marianna Adamo, Roy S Gardner, Andreas Baumbach, Michael Böh, et al. 2021 ESC guidelinesforthe diagnosis and treatment of acute and chronicheartfailure. EuropeanHeartJournal. 2021; 00, 1-128.

5. Luis Manzano Espinosa. Manual práctico de manejo integral del paciente con insuficiencia cardíaca crónica. $4^{\circ}$ edición. Madrid. (2018) ISN 978-84-7867-7678.

6. Grupo de trabajo de ICC y FA de la Sociedad Española de Medicina Interna. Protocolo de manejo de la insuficiencia cardíaca aguda. Disponible en https://www. fesemi.org/grupos/cardiaca/noticias/protocolo-manejo-ica-ver-2021. Comprobado $31 / 08 / 2021$

7. Grupo de trabajo de ICC y FA de la Sociedad Española de Medicina Interna. Nuevo protocolo de manejo de la insuficiencia cardíaca crónica. Disponible en https:// www.fesemi.org/protocololCCronica2021. Comprobado 31/08/2021

8. Grupo de trabajo de ICC y FA de la Sociedad Española de Medicina Interna. Programa UMIPIC. Disponible en https://www.fesemi.org/grupos/cardiaca/umipic/ programa. Comprobado 31/08/2021

9. Cerqueiro JM, González-Franco A, Montero-Pérez-Barquero M, Llácer P, Conde A Dávila MF, Carrera M, et al. Reductioninhospitalisationsandemergencydepartmentvisits for frailpatientswithheartfailure: Resultsofthe UMIPIC healthcareprogramme. RevClinEsp (Barc). 2016; 216(1):8-14.

10. González-Franco A, Cerqueiro JM, Arevalo-Lorido JC, et al. Beneficios de un modelo asistencial integral en pacientes ancianos con insuficiencia cardiaca y elevada comorbilidad: programa UMIPIC. RevClin Esp. 2021. In press. 